TAIWANESE JOURNAL OF MATHEMATICS

Vol. 18, No. 1, pp. 277-283, February 2014

DOI: $10.11650 /$ tjm.18.2014.3405

This paper is available online at http://journal.taiwanmathsoc.org.tw

\title{
BOUNDEDNESS OF COMPOSITION OPERATOR BETWEEN WEIGHTED SPACES OF HOLOMORPHIC FUNCTIONS ON THE UPPER HALF-PLANE
}

\author{
Mohammad Ali Ardalani
}

\begin{abstract}
In this paper, we obtain a necessary and sufficient condition for boundedness of composition operator $C_{\varphi}$, between two different weighted spaces of holomorphic functions on the upper half-plane, whenever one of the weights satisfy certain growth condition.
\end{abstract}

\section{INTRODUCTION}

In Corollary 1.5 of [1], we have characterized boundedness of composition operator $C_{\varphi}$ between two different weighted spaces of holomorphic functions on the upper halfplane. In this paper, with the same assumptions as in [1], we present an alternative necessary and sufficient condition for boundedness of composition operator $C_{\varphi}$, in terms of $\varphi$. Although, we use the concept of associated weight to obtain this characterization, but associated weight does not appear in our characterization (see Theorem 2.3) and this is important because it is difficult to estimate an associated weight. Also, we refer the reader for the analogue problem for weighted spaces on the unit disc to [4] and [5]. We begin with the preliminaries which are required throughout this paper.

Definition 1.1. The sets $\mathbb{D}=\{z \in \mathbb{C}:|z|<1\}$ and $G=\{\omega \in \mathbb{C}: \operatorname{Im} \omega>0\}$ are the unit disc and upper half-plane respectively.

Definition 1.2. A continuous function $v: G \longrightarrow(0, \infty)$ is called a standard weight if $\forall \omega \in G v(\omega)=v(\operatorname{Im} \omega i)$ (i.e $v$ depends only on the imaginary part), $v(s i)<v(t i)$ when $0<s \leq t$ and $\lim _{t \rightarrow 0} v(t i)=0$.

Definition 1.3. Let $v$ be a standard weight on $G$. We say $v$ satisfies condition $(*)$ if there are constants $C, \beta>0$ s.t $\frac{v(t i)}{v(s i)} \leq C\left(\frac{t}{s}\right)^{\beta}$ whenever $0<s \leq t$.

Received June 11, 2013, accepted July 29, 2013.

Communicated by Alexander Vasiliev.

2010 Mathematics Subject Classification: Primary 46E15; Secondary 47B33.

Key words and phrases: Composition operator, Holomorphic functions, Weighted spaces, Upper halfplane. 
It has been proved that $v$ satisfies condition $(*)$ if and only if $\sup _{k \in \mathbb{Z}} \frac{v\left(2^{k+1} i\right)}{v\left(2^{k} i\right)}<\infty$ (see [2]). Indeed, condition $(*)$ states although $v$ is increasing but the speed of its growth is under the control and it can not grow too fast otherwise $\sup _{k \in \mathbb{Z}} \frac{v\left(2^{k+1} i\right)}{v\left(2^{k} i\right)}=\infty$.

Example 1.4. Let $\beta>0$. Then the functions $v_{1}(\omega)=(\operatorname{Im} \omega)^{\beta}$, $v_{2}(\omega)=\min \left(v_{1}(\omega), 1\right), v_{3}(\omega)=\log (\operatorname{Im} \omega+1)$ are standard weights which satisfy condition $(*)$. Also, $v_{2}$ is a bounded weight.

Definition 1.5. Let $O$ be an open subset of $\mathbb{C}$. For a function $f: O \longrightarrow \mathbb{C}$ we define the weighted sup-norm

$$
\|f\|_{v}=\sup _{z \in O}|f(z)| v(z)
$$

and the space

$$
H_{v}(O)=\left\{f: O \longrightarrow \mathbb{C}: f \text { is holomorphic, }\|f\|_{v}<\infty\right\} .
$$

Throughout this paper we deal with the cases $O=\mathbb{D}$ or $O=G$.

Remark 1.6. a. According to a result of Stanev [7], $H_{v}(G) \neq\{0\}$ if and only if there are constants $a, b>0$ such that $v(i t) \leq a e^{b t}, t>0$. we always assume $v$ is such that $H_{v}(G) \neq\{0\}$.

b. For a weight $v$ defined from $\mathbb{D}$ into $(0, \infty)$, we always assume $v$ is radial (i.e $v(z)=v(|z|)$ ), continuous and non-increasing weight with respect to $|z|$.

Definition 1.7. Let $O$ be an open subset of $\mathbb{C}$. Also, suppose $v: O \rightarrow(0,+\infty)$ is a weight. Corresponding to $v$, the associated weight $\tilde{v}$ is defined as follows.

$$
\tilde{v}(z):=\frac{1}{\sup \left\{|f(z)|: f \in H_{v}(O),\|f\|_{v}<1\right\}} \forall z \in O
$$

Remark 1.8. It is easy to see that, always we have $v \leq \tilde{v}$ (see [3]). But in general $v$ and $\tilde{v}$ are not necessarily equivalent( i.e $v$ is not essential). It seems if a standard weight does not satisfy $(*)$, then it is not essential. But this is not really true. As an example, define $v, v_{1}: G \longrightarrow(0, \infty)$ as follows.

$$
\begin{gathered}
v(\omega)= \begin{cases}\frac{1}{1-\ln (\operatorname{Im} \omega)} & \text { if } \operatorname{Im} \omega \leq 1 \\
e^{\operatorname{Im} \omega-1} & \text { if } \operatorname{Im} \omega>1\end{cases} \\
v_{1}(\omega)= \begin{cases}v(\omega) & \text { if } \operatorname{Im} \omega<1 \\
1 & \text { if } \operatorname{Im} \omega \geq 1\end{cases}
\end{gathered}
$$

$v$ is a standard weight which does not satisfy $(*)$. To see $v$ is essential, fix $i t_{0}$ on the imaginary axis. If $t_{0}>1$, put $h_{1}(\omega)=e^{i \omega}$. Hence, $\|f\|_{v}<C$ for some universal 
constant $C$ and $\frac{1}{\left|h_{1}\left(i t_{0}\right)\right|}$ is equivalent to $v\left(i t_{0}\right)$. If $t_{0} \leq 1$, we can find $f \in H_{v_{1}}(G)$ such that $\|f\|_{v_{1}} \leq 1$ and $\frac{1}{\left|f\left(i t_{0}\right)\right|}$ is equivalent to $v_{1}\left(i t_{0}\right)$ since $v_{1}$ is essential. Now, put $h(\omega)=f(\omega) e^{i \omega}$, we have $\|h\|_{v}<C_{1}$ and $\frac{1}{\left|h\left(i t_{0}\right)\right|}$ is equivalent to $v_{1}\left(i t_{0}\right)$ and $v_{1}\left(i t_{0}\right)$ is equivalent to $v\left(i t_{0}\right)$. Therefore, $v$ is essential.

Definition 1.9. Let $v$ be standard weight on $G$. For any $n \in \mathbb{N}$, we define

$$
v_{n}(\omega)=v\left(\frac{4 \operatorname{Im} \omega}{\left(\left|\frac{\omega}{n}+i\right|+\left|\frac{\omega}{n}-i\right|\right)^{2}} i\right) \quad \omega \in G
$$

and

$$
u_{n}(z)=v\left(n \frac{1-|z|}{1+|z|} i\right) \quad z \in \mathbb{D}
$$

Definition 1.10. Define $\alpha: \mathbb{D} \rightarrow \mathbb{C}$ by $\alpha(z)=\frac{1+z}{1-z} i$. An easy computation shows that $\alpha(z)=-2 \frac{\operatorname{Im} z}{|z|^{2}+1-2 \operatorname{Re} z}+\frac{1-|z|^{2}}{|z|^{2}+1-2 \operatorname{Re} z}$. Hence, $\alpha(\mathbb{D}) \subseteq G$. Put $\beta(\omega)=$ $\frac{\omega-i}{\omega+i} \forall \omega \in G$. Then we have $\alpha \circ \beta=i d_{\mid G}$ and $\beta \circ \alpha=i d_{\mid \mathbb{D}}$. Thus $\beta=\alpha^{-1}$ and $\alpha(\mathbb{D})=G$.

Remark 1.11. As we mentioned before, the main result of the present paper (Theorem 2.3) has analogues on the unit disc (see part (a) of Corollary 8 of [4] and PROPOSITION 2.1 of [5]). However if we consider the map $\alpha: \mathbb{D} \rightarrow G$ then $v \circ \alpha$ is non-radial weight on $\mathbb{D}$ and we do not have $\lim _{|z| \rightarrow 1}(v \circ \alpha)(z)=0$. Therefore, it is not possible to derive Theorem 2.3 directly from the corresponding results of radial weights on $\mathbb{D}$.

Remark 1.12. Put $\alpha_{n}(z)=n \frac{1+z}{1-z} i$. Hence $\alpha_{n}: \mathbb{D} \rightarrow G$. Also $\alpha_{n}$ as $\alpha$ is an onto and analytic map. Using relation $|1-z|^{2}=1+|z|^{2}-2 R e z$, it is easy to see that $u_{n}=v_{n} \circ \alpha_{n}$.

Lemma 1.13. Let $v$ be a standard weight on $G$. Then $v_{n}(\omega) \uparrow v(\omega) \omega \in G$ and the map $T: H_{v_{n}}(G) \rightarrow H_{u_{n}}(\mathbb{D})$ defined by $(T f)(z)=f\left(\alpha_{n}(z)\right), z \in \mathbb{D}, f \in H_{v_{n}}(G)$ is an onto isometry i.e $H_{v_{n}}(G)$ is isometrically isomorphic to $H_{u_{n}}(\mathbb{D})$.

Proof. See Proposition 1.4 of [2].

Definition 1.14. Let $\varphi: G \longrightarrow G$ be a holomorphic function. Put $H(O):=$ $\{f \mid f: O \longrightarrow \mathbb{C}$ is holomorphic $\}$. For any $f \in H(O)$ the composition operator $C_{\varphi}: H(O) \longrightarrow H(O)$ defined by $C_{\varphi}(f)=f \circ \varphi$.

Lemma 1.15. Let $v_{1}$ and $v_{2}$ be two standard weights on $G$. Then the following are equivalent:

(i) $C_{\varphi}: H_{v_{1}}(G) \longrightarrow H_{v_{2}}(G)$ is a bounded operator.

(ii) $M:=\sup _{\omega \in G} \frac{v_{2}(\omega)}{\tilde{v}_{1}(\varphi(\omega))}<\infty$

In this case we have $\left\|C_{\varphi}\right\| \leq M$ 
Proof. See Proposition 5 of [6].

\section{Main Result}

To obtain the main theorem of this paper we need the following two lemmas.

Lemma 2.1. For all $n \in \mathbb{N}, \tilde{u}_{n}=\tilde{v}_{n} \circ \alpha_{n}$. Also $\tilde{v}(\omega)=\sup _{n \in \mathbb{N}} \tilde{v}_{n}(\omega), \omega \in G$.

Proof. By the definition of associated weight we have

$$
\tilde{u}_{n}(z)=\frac{1}{\sup \left\{|f(z)|: f: \mathbb{D} \longrightarrow \mathbb{C} \text { is holomorphic, }\|f\|_{u_{n}} \leq 1\right\}}
$$

and

$$
\tilde{v}_{n}(z)=\frac{1}{\sup \left\{|f(z)|: f: G \longrightarrow \mathbb{C} \text { is holomorphic, }\|f\|_{v_{n}} \leq 1\right\}}
$$

Since $T: H_{v_{n}}(G) \rightarrow H_{u_{n}}(\mathbb{D})$ is an onto isometry, $\left\|f \circ \alpha_{n}\right\|_{u_{n}}=\|f\|_{v_{n}}$. This implies that $\left(\tilde{v}_{n} \circ \alpha_{n}\right)(z)=\tilde{u}_{n}(z)$. For the second assertion of the lemma fix $\omega_{0} \in G$. Since

$$
B_{v_{n}}:=\left\{f \in H(G):|f| \leq v_{n} \text { on } G\right\}
$$

is compact subset of $H(G)$ whenever $H(G)$ is endowed with the compact-open topology, using the continuity of point evaluvations yields the supremum in the definition of $\tilde{v}_{n}$ is a maximum ( see also after Definition 1.1 of [3] for similar argument on the unit disc). Therefore, for all $n \in \mathbb{N}$ there is a $f_{n} \in H_{v_{n}}(G)$ with $\left\|f_{n}\right\|_{v_{n}} \leq 1$ such that $\tilde{v}_{n}\left(\omega_{0}\right)=\frac{1}{\left|f_{n}\left(\omega_{0}\right)\right|}$. Since $v_{n} \uparrow v, \frac{1}{v_{n}(\omega)} \leq \frac{1}{v_{1}(\omega)}$. $\left\|f_{n}\right\|_{v_{n}} \leq 1$ implies that for all $n \in \mathbb{N}$ and all $\omega \in G$ we have $\left|f_{n}(\omega)\right| \leq \frac{1}{\left|v_{n}(\omega)\right|} \leq \frac{1}{v_{1}(\omega)}$. Therefore, $\left\{f_{n}\right\}$ is a locally bounded sequence of holomorphic functions on $G$ and Montel's theorem implies that there is a subsequence $f_{n_{k}}$ of $f_{n}$ which converges uniformly to a holomorphic function $f$ on the compact subsets of $G$. $\left\|f_{n}\right\|_{v_{n}} \leq 1$ and $v_{n} \uparrow v$ hence, $\|f\|_{v} \leq 1$ i.e $f \in H_{v}(G)$.

$\tilde{v}_{n_{k}}\left(\omega_{0}\right)=\frac{1}{\left|f_{n_{k}}\left(\omega_{0}\right)\right|} \Rightarrow \lim \tilde{v}_{n_{k}}\left(\omega_{0}\right)=\lim \frac{1}{\left|f_{n_{k}}\left(\omega_{0}\right)\right|}=\frac{1}{\left|f\left(\omega_{0}\right)\right|}$.

Obviously, $\left|f\left(\omega_{0}\right)\right| \leq \sup \left\{\left|g\left(\omega_{0}\right)\right|: g: G \longrightarrow \mathbb{C}\right.$ is holomorphic, $\left.\|g\|_{v} \leq 1\right\}$. Therefore,

$$
\lim _{k \rightarrow \infty} \tilde{v}_{n_{k}}\left(\omega_{0}\right)=\frac{1}{\left|f\left(\omega_{0}\right)\right|} \geq \tilde{v}\left(\omega_{0}\right)
$$

Suppose $v$ and $v^{\prime}$ are two weights such that $v \leq v^{\prime}$. As an immediate consequence of the definition of associated weight we have $\tilde{v} \leq \tilde{v}^{\prime}$. Now, using the fact $v_{n} \uparrow v$, we have

$$
\tilde{v}_{1} \leq \tilde{v}_{2} \leq \ldots \leq \tilde{v}_{n} \leq \ldots \leq \tilde{v}
$$


Therefore,

$$
\sup _{n \in \mathbb{N}} \tilde{v}_{n}\left(\omega_{0}\right) \leq \tilde{v}\left(\omega_{0}\right)
$$

Now, relations (2.1) and (2.2) imply that $\tilde{v}\left(\omega_{0}\right)=\sup _{n} \tilde{v}_{n}\left(\omega_{0}\right)$. Since $\omega_{0} \in G$ is arbitrary proof is complete.

Lemma 2.2. Let $v$ be a standard weight on $G$ satisfying condition (*). Then $\tilde{v}$ is equivalent to

$$
\inf _{k \in \mathbb{N}} \frac{\left\|\left(\frac{\omega-i}{\omega+i}\right)^{k}\right\|_{v}}{\left|\frac{\omega-i}{\omega+i}\right|^{k}}
$$

Proof. Fix $n \in \mathbb{N}$. Obviously, $u_{n}$ is a radial, continuous and non-increasing weight with respect to $|z|$. Also, $\lim _{|z| \rightarrow 1} u_{n}(z)=0$.

Put $\bar{u}_{n}(z):=\frac{1}{\sup _{k \in \mathbb{N}} \frac{|z|^{k}}{\left\|\xi^{k}\right\| u_{n}}}$ where the norm of monomial $\xi^{k}$ is calculated in $H_{u_{n}}(\mathbb{D})$. As in the proof of Lemma 5 in [4]( see line 6 of page 145) we have

$$
u_{n}(z) \leq \tilde{u}_{n}(z) \leq \bar{u}_{n}(z) \leq \max \left(2, \frac{u_{n}(0)}{u_{n}\left(\frac{1}{2}\right)}\right) u_{n}(z)
$$

Relation (2.3) is equivalent to

$$
\frac{1}{\max \left(2, \frac{u_{n}(0)}{u_{n}\left(\frac{1}{2}\right)}\right) u_{n}(z)} \leq \sup _{k \in \mathbb{N}} \frac{|z|^{k}}{\left\|\xi^{k}\right\|_{u_{n}}} \leq \frac{1}{\tilde{u}_{n}(z)}
$$

$\frac{u_{n}(0)}{u_{n}\left(\frac{1}{2}\right)}=\frac{v(n i)}{v\left(\frac{n}{3} i\right)}$. Since, $v$ satisfies condition $(*), \frac{v(n i)}{v\left(\frac{n}{3} i\right)} \leq C\left(\frac{1}{3}\right)^{\beta}=c_{1}$. Thus, $\max \left(2, \frac{u_{n}(0)}{u_{n}\left(\frac{1}{2}\right)}\right) \leq \max \left(2, c_{1}\right)=c_{2}$. Now, inserting this relation in relation (2.4) and using $z$ instead $\xi$ in relation (2.4) we obtain

$$
\frac{1}{c_{2} u_{n}(z)} \leq \sup _{k \in \mathbb{N}} \frac{|z|^{k}}{\left\|z^{k}\right\|_{u_{n}}} \leq \frac{1}{\tilde{u}_{n}(z)}
$$

Using relations $z=\alpha^{-1}(\omega)=\frac{\omega-i}{\omega+i}, u_{n}=v_{n} \circ \alpha_{n}, \tilde{u}_{n}=\tilde{v}_{n} \circ \alpha_{n}$ and surjectivity of $\alpha_{n}$ we have

$$
\frac{1}{c_{2} v_{n}(\omega)} \leq \sup _{k \in \mathbb{N}} \frac{\left|\frac{\omega-i}{\omega+i}\right|^{k}}{\left\|\left(\frac{\omega-i}{\omega+i}\right)^{k}\right\|_{v_{n}}} \leq \frac{1}{\tilde{v}_{n}(\omega)}
$$

Since $v_{n}(\omega) \leq \tilde{v}_{n}(\omega)($ see [4]),

$$
\frac{1}{c_{2} \tilde{v}_{n}(\omega)} \leq \sup _{k \in \mathbb{N}} \frac{\left|\frac{\omega-i}{\omega+i}\right|^{k}}{\left\|\left(\frac{\omega-i}{\omega+i}\right)^{k}\right\|_{v_{n}}} \leq \frac{1}{\tilde{v}_{n}(\omega)}
$$


equivalently,

$$
\tilde{v}_{n}(\omega) \leq \inf _{k \in \mathbb{N}} \frac{\left\|\left(\frac{\omega-i}{\omega+i}\right)^{k}\right\|_{v_{n}}}{\left|\frac{\omega-i}{\omega+i}\right|^{k}} \leq c_{2} \tilde{v}_{n}(\omega)
$$

Now, by taking supremum over $n \in \mathbb{N}$ in relation (2.5), considering the relation $v_{n} \uparrow v$ and applying the second assertion of Lemma 2.1, we obtain

$$
\tilde{v}(\omega) \leq \inf _{k \in \mathbb{N}} \frac{\left\|\left(\frac{\omega-i}{\omega+i}\right)^{k}\right\|_{v}}{\left|\frac{\omega-i}{\omega+i}\right|^{k}} \leq c_{2} \tilde{v}(\omega)
$$

which proves the lemma.

Theorem 2.3. Let $v_{1}$ and $v_{2}$ be two standard weights on $G$ such that $v_{1}$ satisfies $(*)$. Then the following are equivalent.

(i) $C_{\varphi}: H_{v_{1}}(G) \longrightarrow H_{v_{2}}(G)$ is a bounded operator.

(ii) $M:=\sup _{k \in \mathbb{N}} \frac{\left\|\left(\frac{\varphi(\omega)-i}{\varphi(\omega)+i}\right)^{k}\right\|_{v_{2}}}{\left\|\left(\frac{\omega-i}{\omega+i}\right)^{k}\right\|_{v_{1}}}<\infty$

In this case we have $\left\|C_{\varphi}\right\| \leq M$.

Proof. Lemma 1.15 implies that (i) is equivalent to

$$
M:=\sup _{\omega \in G} \frac{v_{2}(\omega)}{\tilde{v}_{1}(\varphi(\omega))}<\infty
$$

Now, using Lemma 2.2 we have

$$
\tilde{v}_{1}(\omega) \sim \inf _{k \in \mathbb{N}} \frac{\left\|\left(\frac{\omega-i}{\omega+i}\right)^{k}\right\|_{v_{1}}}{\left|\frac{\omega-i}{\omega+i}\right|^{k}}
$$

which implies that

$$
\frac{1}{\tilde{v}_{1}(\varphi(\omega))} \sim \sup _{k \in \mathbb{N}} \frac{\left|\frac{\varphi(\omega)-i}{\varphi(\omega)+i}\right|^{k}}{\left\|\left(\frac{\omega-i}{\omega+i}\right)^{k}\right\|_{v_{1}}}
$$

note that $\varphi$ does not appear in the denominator of the right hand side of relation (2.6) since it is a monomial in $H_{v_{1}}(G)$ which does not depend on $\tilde{v}_{1}$.

Therefore, (i) is equivalent to

$$
\sup _{\omega \in G} v_{2}(\omega) \sup _{k \in \mathbb{N}} \frac{\left|\frac{\varphi(\omega)-i}{\varphi(\omega)+i}\right|^{k}}{\left\|\left(\frac{\omega-i}{\omega+i}\right)^{k}\right\|_{v_{1}}}=\sup _{k \in \mathbb{N}} \frac{\left\|\left(\frac{\varphi(\omega)-i}{\varphi(\omega)+i}\right)^{k}\right\|_{v_{2}}}{\left\|\left(\frac{\omega-i}{\omega+i}\right)^{k}\right\|_{v_{1}}}<\infty
$$

Remark 2.4. Looking at page 147 of [4], one can see the analogue of Theorem 2.3 is true for weighted spaces of $H_{v}(O)$ whenever $O=\{z \in \mathbb{C}: \operatorname{Re} z>0\}$ and our weights are bounded, continuous, depending only on the real part and tending to zero at imaginary axis. It is worth to be worked out, is it possible to extend this result of [4] to unbounded weights by using the method we have presented here? 


\title{
ACKNOWLEDGMENT
}

The author would like to thank the referee for her/his valuable suggestions.

\section{REFERENCES}

1. M. A. Ardalani and W. Lusky, Bounded operators on weighted spaces of holomorphic functions on the upper half-plane, Studia Math., 209 (2012), 225-234.

2. M. A. Ardalani and W. Lusky, Weighted spaces of holomorphic functions on the upper half-plane, Math. Scandinavia, 111 (2012), 244-260.

3. K. Bieirstedt, J. Bonet and J. Taskinen, Associated weights and spaces of holomorphic functions, Studia Math., 127(2) (1988), 137-168.

4. J. Bonet, P. Domanski and M. Lindstrom, Essential norm and weak compactness of composition operators on weighted Banach spaces of analytic functions, Bull. Canad. Math., 42 (1999), 139-148.

5. J. Bonet, P. Domanski, M. Lindstrom and J. Taskinen, Composition operators between weighted Banach spaces of analytic functions, J. Austral. Math. Soc. (Series A), 64(1) (1998), 101-118.

6. J. Bonet, M. Friz and E. Jorda, Composition operators between weighted inductive limits of spaces of holomorphic functions, Publ. Math. Debercen 67, 3-4 (6), 2005.

7. M. A. Stanev, Weighted Banach spaces of holomorphic functions in the upper half-plane, arXiv:math.FA/9911082 vl, 1999.

\author{
Mohammad Ali Ardalani \\ Department of Mathematics \\ Faculty of Science \\ University of Kurdistan \\ Pasdaran Ave. \\ 66177-175 Sanandaj \\ Iran \\ E-mail: M.Ardalani@uok.ac.ir
}

